\title{
Minimal important difference and responsiveness of 2-minute walk test performance in people with COPD undergoing pulmonary rehabilitation
}

This article was published in the following Dove Press journal:

International Journal of COPD

9 October 2017

Number of times this article has been viewed

\author{
Kylie Nicole Johnston \\ Adrian James Potter \\ Anna Caroline Phillips \\ School of Health Sciences, Sansom \\ Institute for Health Research, \\ University of South Australia, \\ Adelaide, SA, Australia
}

Correspondence: Kylie Nicole Johnston School of Health Sciences, Sansom Institute for Health Research, University of South Australia, City East Campus, Corner North Terrace and Frome Road, Adelaide, SA 5000, Australia

$\mathrm{Tel}+6 \mathrm{I} 883022086$

Fax +6I 883022853

Email kylie.johnston@unisa.edu.au
Background and objectives: Field exercise tests (eg, 6-minute walk test [6MWT]) are important measures of functional exercise capacity in people with COPD. Shorter tests such as the 2-minute walk test (2MWT) may offer advantages in some populations but lack information about responsiveness to change. This study examined responsiveness, minimal important difference (MID), test-retest reliability, and construct validity of the 2MWT in people with stable COPD attending outpatient pulmonary rehabilitation (PR).

Methods: At pre-PR assessment, study participants completed a $2 \mathrm{MWT}$ twice in addition to usual measures (6MWT and Chronic Respiratory Questionnaire). At post-PR assessment following a standard PR program, measures were repeated and global rating of change scores obtained (patient and therapist). Pre-post program change scores were examined for correlations with change in 2-minute walk distance (2MWD) and used (where $r \geq 0.3$ ) to estimate the MID through anchor-based methods. Distribution-based estimates based on standard error of measurement were examined. Test-retest reliability (intraclass correlation coefficient [ICC] and Bland-Altman agreement) and validity (Pearson correlation with 6-minute walk distance [6MWD]) were reported.

Results: Fifty-nine people (28 men) with stable COPD, mean age 68 years (SD 10 years), and percentage predicted forced expiratory volume in 1 second $48 \%$ (SD 20\%) attended pre-PR assessment. Test-retest ICC for same-session 2MWD was 0.985. A mean difference of $2.4 \mathrm{~m}$ (95\% confidence interval $[\mathrm{CI}] 0.7-4.0 \mathrm{~m}, P=0.006$ ) occurred between the first and second trials. 2MWD and 6MWD were highly correlated $(r=0.87, P<0.001)$. Forty-one participants completed $\mathrm{PR}$ and were included in responsiveness and MID analysis. Mean 2MWD improved significantly post-PR $(8.8 \mathrm{~m}, 95 \%$ CI $3.6-14 \mathrm{~m}, P=0.001)$. The MID in $2 \mathrm{MWD}$, anchored against clinically meaningful change in $6 \mathrm{MWD}$, was $5.5 \mathrm{~m}$ (area under curve $=0.81, P=0.001$ ). Distribution-based methods estimated an MID of $4 \mathrm{~m}$.

Conclusion: Change in 2MWD of at least $5.5 \mathrm{~m}$ following a PR program corresponded to a clinically meaningful change. A practice test is recommended due to learning effects.

Keywords: exercise test, psychometrics, COPD, rehabilitation

\section{Introduction}

Field exercise tests based on walking are used to assess functional exercise capacity in people with chronic lung disease and to prescribe intensity of exercise training. The 6-minute walk test (6MWT), incremental shuttle walk test (ISWT), and endurance shuttle walk test are the three most established field tests in this patient population with accepted reliability, validity, and responsiveness to exercise training interventions in stable patients. ${ }^{1}$ 
In some situations, there may be barriers to the implementation of these tests associated with ability of the patient, acceptability, time, and resource restraints. Due to learning effects, the use of repeat testing for the 6MWT and ISWT is recommended, ${ }^{2}$ although not yet widely implemented in the clinical setting. Recent audit data of 210 pulmonary rehabilitation (PR) programs in England and Wales indicated that a repeat walking test was conducted at initial assessment in a minority $(22 \%)$ of cases. $^{3}$

When utilized during a hospital admission for acute exacerbation of COPD, the 6MWT showed wide variability between the first two trials ${ }^{4}$ and confirmed the need for a repeat test in this population. However, only $19 \%$ participated in the second test and this may indicate the low feasibility of 6MWT repeat testing in the acute setting. ${ }^{4}$ As total time spent walking during admission for an exacerbation of COPD has been recorded by accelerometer as 7.2 minute/day, ${ }^{5}$ same-day repetition of the 6MWT may be unacceptable to patients.

The 2-minute walk test (2MWT) may be more feasible in some settings. Frail elderly patients undergoing geriatric inpatient rehabilitation were unable to complete a single trial of the $6 \mathrm{MWT}$ but were better able to tolerate the $2 \mathrm{MWT}{ }^{6}{ }^{6}$ The 2MWT has been utilized as a measure of functional exercise capacity in patients before and after cardiac surgery, ${ }^{7}$ in patients with inflammatory muscle disorders, ${ }^{8}$ and in people with chronic graft versus host disease. ${ }^{9}$

Few studies to date have reported on measurement properties of the 2MWT in people with COPD. Two studies examined reliability of same-day repeat testing in people with stable COPD and described low within-subject variability $(5.1 \%),{ }^{10}$ high intraclass correlation coefficient (ICC) (0.9994), ${ }^{11}$ and mean differences between up to three trials ranging from $0.3 \mathrm{~m}^{11}$ to $3.8 \mathrm{~m} .{ }^{10}$ Correlations of 2-minute walk distance (2MWD) with 6-minute walk distance (6MWD) $(r=0.937, P<0.001, \mathrm{n}=45)$ and peak oxygen uptake during a maximal cardiopulmonary exercise test $(r=0.555$, $P<0.001, \mathrm{n}=45)^{11}$ support validity as a measure of exercise capacity. Nadir values for oxygen saturation detected during 2MWT and 6MWT in people with severe COPD $(n=26)$ are also closely correlated $(r=0.81) .{ }^{12}$

Responsiveness of the 2MWT to change in people with COPD has been examined after delivery of bronchodilator medication where standardized mean response (mean change/standard deviation of change) was 0.75 , lower than responsiveness to same in the $6 \mathrm{MWT}(0.84) .{ }^{10}$ Leung et al ${ }^{11}$ reported large standardized mean responses for the 2MWD and 6MWD after an inpatient PR program (1.25 and 1.70, respectively), although sample size was small $(n=9)$ and did not include six participants who dropped out.

Further information about measurement properties of the 2MWT in people with COPD is required to determine clinical usefulness of this test in this population. The primary aim of this study was to examine responsiveness of the 2MWT in stable patients with COPD attending outpatient PR and to determine the minimal important difference (MID) using anchor and distribution-based methods. Secondary aims were to examine the test-retest reliability and construct validity of the 2MWT. Our hypotheses were that 2MWD would show similar responsiveness following outpatient PR to the 6MWD, high test-retest reliability, and high construct validity with the $6 \mathrm{MWT}$.

\section{Methods \\ Design and participants}

People with stable COPD confirmed by spirometry ${ }^{13}$ were recruited from an outpatient PR program. Participants were excluded if they had severe comorbidities that prevented exercise training (severe cardiac, neurological, or musculoskeletal conditions) or if they met any of the absolute contraindications to conduct of a 6MWT. ${ }^{2}$ Ethical approval was granted prior to study commencement by the Royal Adelaide Hospital Human Research Ethics Committee (protocol no 141205, reference no HREC/14/RAH/541) and the University of South Australia's Human Research Ethics Committee (application no 0000033829). All participants provided written informed consent prior to their commencement in this study.

\section{PR program and outcome measures}

Data collection took place before and after an 8-week PR program (Figure 1). The PR program followed a standard protocol of twice weekly supervised sessions for 8 weeks. Sessions consisted of an individually prescribed exercise program conducted in a group setting $(2 \times 1$ hour/week), followed by group education and self-management sessions ( $1 \times 1$ hour/week). Demographic information including age, living arrangements, smoking status, mobility aids, and disease-specific information such as lung function, comorbidities, and medications were obtained from the participants' medical records. Pre- and post-PR measures were conducted by program staff according to published procedures ${ }^{2}$ including a single 6MWT (30 m straight track) and completion of the interviewer-administered version of the Chronic Respiratory Questionnaire (CRQ). ${ }^{14}$

After completing the rehabilitation program and prior to any walking test, participants were asked to rate their change 


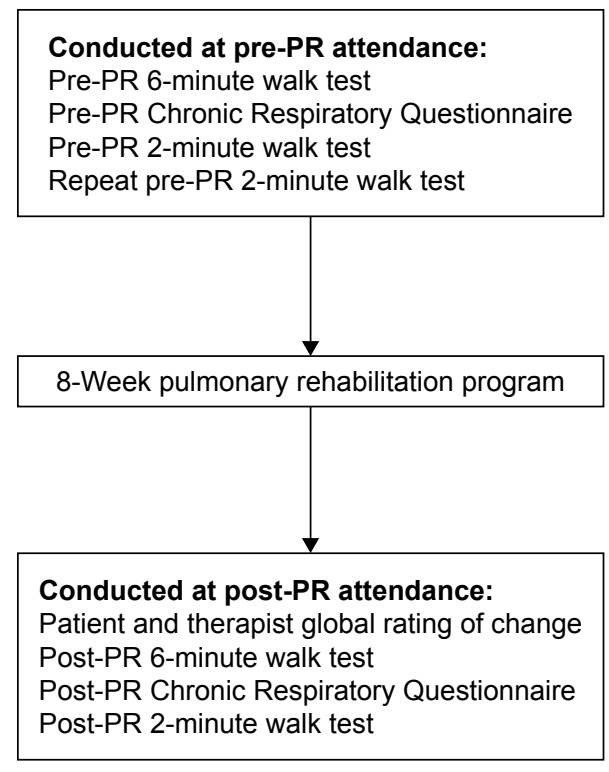

Figure I Process flow of measures recorded. Abbreviation: PR, pulmonary rehabilitation.

in walking ability on a global rating of change (GRC) scale by a study investigator not involved with delivery or assessment of the PR program. This method is recommended to determine change within an individual in MID studies. ${ }^{15,16}$ Participants were asked "Has there been any change in your walking ability since you started the PR program?", and responses were invited according to a Likert scale ( -7 , very great deal worse; 0 , no change; +7 , very great deal better). A therapist who supervised the PR program was asked to make an independent GRC rating regarding the participant's walking ability using the same scale.

The 2MWT was conducted by the study investigators twice at pre-PR assessment and once following completion of the PR program. All participants rested for at least 30 minutes after a $6 \mathrm{MWT}$ or 15 minutes after a $2 \mathrm{MWT}$ beyond the return of pulse oximetry, heart rate, and dyspnea to baseline measures before a subsequent 2MWT was conducted.

Conduct of the 2MWT followed a protocol modified from guidelines for the $6 \mathrm{MWT}^{2}$ with standardized instructions and encouragement every 30 seconds. Participants were asked to walk as far as they could in 2 minutes, up and back along a straight track of $30 \mathrm{~m}$, with the total distance walked recorded. Pulse oximetry and heart rate were measured continuously. Participant-reported dyspnea and leg fatigue assessed using modified Borg scores were recorded at baseline and at test completion. Criteria for test cessation were the same as those recommended for the $6 \mathrm{MWT}^{2}$ (pulse oximetry $<80 \%$, chest pain, intolerable dyspnea, leg cramps, staggering, diaphoresis, and pale appearance).

\section{Data analysis}

Characteristics of participants were expressed as relative frequencies, mean (SD), or median (interquartile range [IQR]). Comorbidities were determined using a pharmaceuticalbased index ${ }^{17}$ which includes 42 comorbidities as determined by World Health Organization Anatomical and Therapeutic Chemical classification. ${ }^{18}$

Test-retest reliability of the 2MWT was estimated by calculation of the ICC (two-way random-effects model and absolute agreement). A paired $t$-test was performed to assess any learning effect between test and retest observations, and a Bland-Altman plot constructed to describe $95 \%$ limits of agreement. To examine construct validity, Pearson's correlation was used to quantify the association between best (highest) pre-PR 2MWD and pre-PR 6MWD. The 6MWT is an established measure of functional exercise capacity, ${ }^{2}$ and the 2MWT was hypothesized to measure a similar construct. To identify differences in physiological responses between the 2MWT and 6MWT, paired $t$-tests or Wilcoxon signed rank tests (for non-normally distributed data) were performed for the measurements of nadir $\mathrm{SpO}_{2}$, end test heart rate, and end test modified Borg dyspnea scores.

Response to the PR program was evaluated using paired $t$-tests between pre- and post measurements of the 2MWD (first tests pre- and post-PR), 6MWD, and CRQ domains. Effect sizes for these measures were calculated using the formula $d=\left(\right.$ mean $_{\text {post }}-$ mean $\left._{\text {pre }}\right) / \mathrm{SD}_{\text {pooled }}{ }^{19}$ External responsiveness of the 2MWT was examined through Pearson correlation analysis between change in 2MWD (first tests pre- and post-PR) and change in 6MWD following PR.

A range of potential anchors were examined for suitability in estimation of an anchor-based MID. ${ }^{20,21}$ These were GRC score (patient and therapist), change in 6MWD and change in CRQ domain scores. To determine suitability for use as an anchor, the correlation with change in 2MWD was examined and those where correlation was $r \geq 0.3$ were considered suitable and used in further analysis. ${ }^{21}$ The sensitivity and specificity for change in the 2MWD to discriminate between individuals who had been classified as "changed" and "unchanged" was calculated, and a receiver operating characteristic (ROC) curve obtained for appropriate anchor measures. Planned cut points for changed and unchanged were established for GRC scores (GRC score $\geq 2 /$ GRC score $<2$ ). Planned cut points for 6MWD and CRQ were based on established MID in these measures of 0.5 points ${ }^{22}$ and $25 \mathrm{~m},{ }^{16}$ respectively. The data point closest to the upper left corner of the ROC curve corresponded to the MID. Area under the curve (AUC) values 
and $95 \%$ confidence intervals (CIs) were obtained for each ROC curve.

For distribution-based MID, standard error of measurement (SEM) was calculated according to the formula $\mathrm{SEM}=\sigma_{1} \sqrt{ }(1-r)$ where $\sigma_{1}$ is the baseline standard deviation and $r$ is the test-retest reliability. ${ }^{23}$

Statistical analyses were performed using the Statistical Package for Social Sciences Version 22 software (IBM Corporation, Armonk, NY, USA). A $P$-value of $<0.05$ was considered statistically significant.

\section{Results}

\section{Participants}

Seventy-two participants were recruited and attended pre-PR assessment. Of these, 13 participants subsequently declined a repeat $2 \mathrm{MWT}$. This resulted in 59 participants available for the analysis of test-retest reliability and construct validity. Characteristics of this sample are shown in Table 1. Common comorbidities included gastric acid disorder (43\%), hypertension (40\%), chronic heart failure (36\%), hyperlipidemia (28\%), depression (28\%), arrhythmia (21\%), musculoskeletal pain $(19 \%)$, diabetes $(16 \%)$, and anxiety $(14 \%)$.

\section{Test-retest reliability of the $2 M W D$}

The 2MWD showed excellent test-retest reliability with an ICC of 0.985 (95\% CI 0.972-0.992). There was a mean difference of $2.4 \mathrm{~m}(95 \% \mathrm{CI} 0.7-4.0 \mathrm{~m}, P=0.006)$ between $2 \mathrm{MWD}$ in the first (mean $[\mathrm{SD}]=129.2$ [28.0] $\mathrm{m}$ ) and second (131.6 [27.3] m) trials. A Bland-Altman plot (Figure 2) demonstrated $95 \%$ limits of agreement for the difference between first and second tests of $-10.1 \mathrm{~m}$ to $14.8 \mathrm{~m}$.

\section{Construct validity of the 2MWT}

Values of best 2MWD pre-PR and 6MWD pre-PR were highly correlated $(r=0.87, P<0.001)$. Comparison of physiological responses between the best $2 \mathrm{MWT}$ and $6 \mathrm{MWT}$ indicated no difference between nadir $\mathrm{SpO}_{2}$ values (90 [5] and 90 [5], respectively, mean difference $=-0.4,95 \% \mathrm{CI}$ of difference -1.4 to 0.6 ). End test heart rate and modified Borg Dyspnea scores were greater for the 6MWT than for the 2MWT (heart rate 105 [15] vs 100 [20] beats/min, $P=0.03$; modified Borg Dyspnea 3.6 [1.9] vs 3.0 [1.5], $P=0.005)$.

\section{Responsiveness and MID of the 2MWD}

Following the PR program, 46 (64\%) of the 72 recruited patients participated in post-PR assessment. Reasons that participants did not complete post-PR assessment included that they never started $(n=5)$ or did not complete PR $(n=14)$;
Table I Pre-PR assessment characteristics of participants included in reliability and validity analyses $(n=59)$

\begin{tabular}{|c|c|}
\hline Characteristics & $\begin{array}{l}\text { Mean (SD) or } \\
\text { frequency (\%) }\end{array}$ \\
\hline Age (years) & $68.4(9.8)$ \\
\hline Males & $28(48 \%)$ \\
\hline Body mass index $\left(\mathrm{kg} / \mathrm{m}^{2}\right)$ & $26.7(5.9)$ \\
\hline Number of pharmacologically & $3(2)$ \\
\hline \multicolumn{2}{|l|}{ managed comorbidities } \\
\hline \multicolumn{2}{|l|}{ Living arrangements } \\
\hline Home alone & $25(42 \%)$ \\
\hline Home with spouse/partner & $22(37 \%)$ \\
\hline Home with child/grandchild & $8(14 \%)$ \\
\hline Home with friend/other & $3(5 \%)$ \\
\hline Residential care & I (2\%) \\
\hline FEV \%pred & $47.8(20.2)$ \\
\hline $\mathrm{FEV}_{1} / \mathrm{FVC}$ & $45.0(15.0)$ \\
\hline \multicolumn{2}{|l|}{ Smoking status } \\
\hline Never smoked & $5(9 \%)$ \\
\hline Current smoker & $12(20 \%)$ \\
\hline Exsmoker & 42 (7।\%) \\
\hline Long-term oxygen therapy & $7(12 \%)$ \\
\hline Gait aid used during walking tests & $6(10 \%)$ \\
\hline 6MWD (m) & $330.5(96.0)$ \\
\hline Best 2MWD (m) & I32.5 (27.3) \\
\hline \multicolumn{2}{|l|}{ CRQ $n=55$} \\
\hline CRQ-dyspnea & $3.31(1.23)$ \\
\hline CRQ-emotional function & $4.44(1.35)$ \\
\hline CRQ-mastery, median (IQR) & $4.50(2.5)$ \\
\hline CRQ-fatigue & $3.65(1.31)$ \\
\hline \multicolumn{2}{|l|}{$\mathrm{mMRC}$ scale $\mathrm{n}=57$} \\
\hline Grade 0 & $3(5 \%)$ \\
\hline Grade I & $16(28 \%)$ \\
\hline Grade 2 & $21(37 \%)$ \\
\hline Grade 3 & $12(21 \%)$ \\
\hline Grade 4 & $5(9 \%)$ \\
\hline
\end{tabular}

Notes: Grade 0, I only get breathless with strenuous exercise; Grade I, I get short of breath when hurrying on level ground or walking up a slight hill; Grade 2, on level ground, I walk slower than people of the same age because of breathlessness, or I have to stop for breath when walking at my own pace on the level; Grade 3, I stop for breath after walking $\sim 100 \mathrm{~m}$ or after a few minutes on level ground; Grade 4, I am too breathless to leave the house or I am breathless when dressing or undressing.

Abbreviations: CRQ, Chronic Respiratory Questionnaire; FEV , forced expiratory volume in I second; FEV \%pred, percentage predicted $\mathrm{FEV}$; FVC, forced vital capacity; IQR, interquartile range; 2MWD, 2-minute walk distance; 6MWD, 6-minute walk distance; mMRC, modified Medical Research Council; PR, pulmonary rehabilitation.

completed PR but did not attend post-PR assessment $(n=3)$ or staff unavailability $(n=4)$. Post-PR data were not analyzed for five participants who were not well with an acute exacerbation during the PR program $(n=4)$ or on the day of post-PR assessment $(n=1)$, resulting in a sample of 41 participants for the analysis of 2MWT responsiveness. Those who did not complete valid post-PR testing included a higher proportion of current smokers and had lower percentage predicted forced expiratory volume in 1 second (FEV ${ }_{1}$ \%pred) (Table S1). 


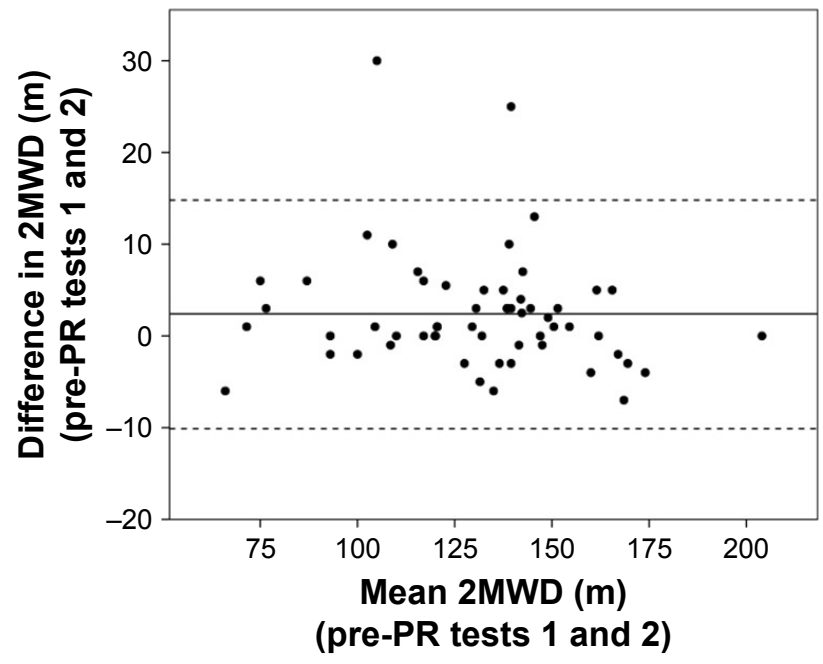

Figure 2 Bland-Altman plot for test-retest reliability of 2MWD, with difference between measurements ( $y$-axis) plotted against mean of the measurements ( $x$-axis). Notes: The central horizontal line represents the mean bias $(2.4 \mathrm{~m})$; the lower and upper dashed horizontal lines represent the $95 \%$ limits of agreement $(-10.1$ and $14.8 \mathrm{~m})$.

Abbreviations: 2MWD, 2-minute walk distance; PR, pulmonary rehabilitation.

Mean 2MWD improved significantly post-PR with a mean difference of $8.8 \mathrm{~m}$ and effect size of 0.3 (Table 2). Significant improvements were also observed in 6MWD and CRQ domains of emotional function and fatigue (Table 2). The median GRC score for participants was +4 ("moderately better", IQR $=2$, range -2 to +7 ) and the median GRC score for therapists was +3 ("somewhat better", IQR $=2$, range -2 to +5 ).

A moderate correlation $(r=0.5, P=0.001)$ was observed between change in $2 \mathrm{MWD}$ and change in 6MWD. However, no significant correlations existed between patient GRC score and change in either 2MWD or 6MWD $(r=-0.19$, $P=0.24$ and $r=0.01, P=0.94)$, or with change in $2 \mathrm{MWD}$ and therapist GRC ( $r=-0.10, P=0.53)$ or any domains of the CRQ (dyspnea $r=0.01, P=0.96$; emotional function $r=-0.14, P=0.40$; mastery $r=-0.26, P=0.13$, fatigue $r=0.08$,
$P=0.64)$. A change in $2 \mathrm{MWD}$ of $5.5 \mathrm{~m}$ (the anchor-based MID) had the best combination of sensitivity (0.76) and specificity (0.74) for identifying participants who achieved the minimum clinically important change in 6MWD of at least $25 \mathrm{~m},{ }^{16}$ with AUC of 0.81 (95\% CI 0.66-0.95, $P=0.001$ ). Defining responders as achieving at least the MID postprogram ( $25 \mathrm{~m}$ for the $6 \mathrm{MWT}$ and $5.5 \mathrm{~m}$ for the $2 \mathrm{MWT})$, there was agreement between the two walking tests in identifying the same participants as responders (16 participants) and nonresponders (14 participants) in $75 \%$ of cases. Five $(12.5 \%)$ participants were responders in the 6MWT but not in the 2MWT, and another five (12.5\%) participants were responders in the 2MWT but not in the 6MWT. Using the baseline standard deviation for the 2MWD and the calculated ICC of 0.985 , the distribution-based estimate for MID in 2MWD was $4.0 \mathrm{~m}$.

No walking tests in any part of the study were terminated due to adverse events.

\section{Discussion}

This study estimated the MID in 2MWD for people with COPD following an outpatient PR program to be between $5.5 \mathrm{~m}$ (anchor-based estimate) and $4.0 \mathrm{~m}$ (distribution-based estimate). 2MWD and 6MWD showed similar responsiveness to PR demonstrated through equivalent effect sizes. As the first study to report an MID for the 2MWD in any clinical population, this addresses a gap identified in a recent systematic review of the measurement properties of this test. ${ }^{24}$ Previous studies have calculated a minimal detectable change at the $95 \%$ confidence level for 2 MWD based on the SEM in adults following knee arthroplasty, ${ }^{25}$ stroke, ${ }^{26}$ or poliomyelitis, ${ }^{27}$ which indicate the extent of change expected from statistical variation. However, guidance about change linked to a meaningful outcome derived from a patient-related anchor is considered more clinically relevant. ${ }^{21,28}$

Table 2 Outcome measure responses to $P R, n=4 I^{a}$

\begin{tabular}{|c|c|c|c|c|c|}
\hline Measure & $\begin{array}{l}\text { Pre-PR, } \\
\text { mean (SD) }\end{array}$ & $\begin{array}{l}\text { Post-PR, } \\
\text { mean (SD) }\end{array}$ & $\begin{array}{l}\text { Change post-pre-PR } \\
(95 \% \mathrm{Cl})\end{array}$ & $\begin{array}{l}\text { Effect } \\
\text { size }\end{array}$ & $P$-value \\
\hline 2MWD (m) & $125.4(32.3)$ & $134.2(28.5)$ & 8.8 (3.6 to 14.0$)$ & 0.3 & 0.001 \\
\hline 6MWD (m) & $324.8(97)$ & $350.9(107)$ & $26.0(0.6$ to $5 \mathrm{I})$ & 0.3 & 0.045 \\
\hline CRQ-D & $3.40(1.37)$ & $3.57(1.23)$ & $0.17(-0.22$ to 0.56$)$ & 0.1 & 0.39 \\
\hline CRQ-EF & $4.76(1.27)$ & $5.24(1.40)$ & 0.47 (0.06 to 0.87$)$ & 0.4 & 0.03 \\
\hline CRQ-M & $5.11(2.38)$ & $5.20(1.31)$ & $0.08(-0.65$ to 0.81$)$ & 0.1 & 0.82 \\
\hline CRQ-F & $3.97(1.26)$ & $4.60(1.44)$ & 0.64 (0.23 to I.04) & 0.5 & 0.003 \\
\hline
\end{tabular}

Notes: Significant at $P<0.05$ level. ${ }^{a} n=4$ I for $2 M W D ; n=40$ for $6 M W D, n=37$ for CRQ domains.

Abbreviations: $\mathrm{Cl}$, confidence interval; CRQ-D, Chronic Respiratory Questionnaire dyspnea domain; CRQ-EF, Chronic Respiratory Questionnaire emotional function domain; CRQ-M, Chronic Respiratory Questionnaire mastery domain; CRQ-F, Chronic Respiratory Questionnaire fatigue domain; 2MWD, 2-minute walk distance; 6MWD, 6-minute walk distance; PR, pulmonary rehabilitation. 
We established the cut-point for participants being changed or unchanged in walking ability based on them achieving at least $25 \mathrm{~m}$ difference in 6MWD after PR. This was based on the MID for the 6MWD established in an Australian study after implementation of a comparable PR program. ${ }^{16}$ This value is also consistent with the average of available anchor-based estimates for change in 6MWD in chronic respiratory disease (range 21.6-38.6 with median $24.8 \mathrm{~m})^{1}$ and within the range reported by a review of $6 \mathrm{MWD}$ MID in adults with medical conditions $(14.0-30.5 \mathrm{~m}) .^{29}$ While change in 2MWD in this study was moderately correlated with change in $6 \mathrm{MWD}$, neither walking distance was related to participant or therapist-reported GRC in walking ability or CRQ measures. A review of the literature by Singh et $\mathrm{al}^{1}$ found that from six included studies evaluating the MID of the 6MWD in people with COPD, three studies did not provide anchor-based estimates, in some cases due to low correlations of changes with patient-reported anchors including the CRQ, the St George's Respiratory Questionnaire, and the Feeling Thermometer. ${ }^{30}$ Significant correlation with GRC scores in walking ability was found in a study by Holland et al, ${ }^{16}$ which enabled these investigators to determine the MID for the 6MWD based on this indicator. In our study, lack of correlations of change in other measures with change in 2MWD may have resulted from the low magnitude of changes observed in most outcomes, especially for dyspnea and mastery domains of the CRQ. While changes in CRQ domains of emotional function and fatigue were moderate, these could have been associated with benefits experienced by the participants in aspects of health and wellbeing other than walking ability.

GRC scores aim to identify the magnitude of difference that is noticeable to the patient and are considered to have high face validity, ${ }^{31}$ but there are clinical research reports of mismatch between GRC and observed change. ${ }^{32,33}$ One explanation for this paradox is evidence that patient estimates of change in their condition are heavily influenced by their current status..$^{34,35}$ Using data from seven clinical studies in patients with musculoskeletal disorders, Kamper et $\mathrm{al}^{35}$ found that, while change scores for pain and disability were associated with GRC scores, postintervention scores nearly always correlated more strongly with GRC. Their interpretation was that "rating of change" scores actually reflect current state rather than truly reflecting change. Exploration of our data found evidence of a similar trend, with post-PR 2MWD and post-PR 6MWD both significantly correlated with patient GRC ( $r=0.4, P=0.017$ and $r=0.4, P=0.005$, respectively). Similar relationships were also seen in our pre-PR measures, with correlations between GRC scores (reported after PR) and baseline measures of pre-PR 2MWD ( $r=0.4, P=0.006)$ and pre-PR $6 \mathrm{MWD}(r=0.5, P=0.001)$. It is possible that participants in our study may have reflected on their current or previous walking status but not on change in their walking ability in response to the GRC scale.

This study confirms that the 2MWT is a reliable measure and provides further evidence of a learning effect between the first and second trials. In this study, the two trials of 2MWT were conducted at least 30 minutes after completion of a 6MWT on the same track. It is not known whether mean difference between the 2MWT trials would have been even greater if the participants had not also experienced the longer walking test at the same testing occasion. However, the difference between the first and second 2MWD trials in our study $(2.4 \mathrm{~m})$ was similar to that reported by Eiser et a ${ }^{10}$ $(2.5 \mathrm{~m})$ who did not conduct a 6MWT, suggesting that the learning effect was not influenced by the prior 6MWT. The 2MWD correlated highly with 6MWD, supporting its construct validity as a measure of functional exercise capacity. The 2MWT demonstrated statistically lower end test measures of heart rate and perceived dyspnea than the $6 \mathrm{MWT}$, but these differences were less than the reported variations in repeat end 6MWT heart rate $(-4 \text { to }+8 \mathrm{bpm})^{1}$ and for the modified Borg Dyspnea score, less than the reported minimally clinically important difference of 1 unit. ${ }^{36}$

\section{Study limitations}

Participants from this study were recruited from a single center, limiting the generalizability of results. This study was limited by the conduct of only one 6MWT pre- and post-PR, which was the usual practice at this center. In our examination of responsiveness of the 2MWD to PR, the impact of this was minimized by comparing only the first trials of each test before and after PR. Baseline 6MWD recorded in this study sample (mean $330.5 \mathrm{~m}$ ) was low compared to reports in other Australian programs where two preprogram 6MWTs were conducted in both hospital (mean baseline 6MWD $=359 \mathrm{~m}^{16}$ ) and community settings (mean baseline 6MWD $=365 \mathrm{~m}^{37}$ ), although samples are not equivalent to this study in characteristics such as $\mathrm{FEV}_{1}$ \%pred, comorbidities, and PR program inclusions/ exclusions. In contrast, baseline 6WMD in this study was similar to end-program 6MWD reported in an audit of 210 PR programs across England and Wales (median discharge $6 \mathrm{MWD}=330 \mathrm{~m}, \mathrm{n}=1,720^{3}$ ). The use of only one 6MWT pre-PR may have also resulted in prescription of a lower than optimal walking training intensity and reduced the overall effectiveness of the PR program. For this or other reasons, the PR program at this center resulted in only 
small effect sizes for both functional exercise tolerance and health-related quality of life measures in our study sample, some of which did not reach statistical significance. Programs using similar training intensity have demonstrated greater postprogram improvements in 6MWD (eg, mean changes of $44 \mathrm{~m}^{37}$ to $66 \mathrm{~m}^{16}$ reported) than observed in this study $(26 \mathrm{~m})$, along with greater changes in health-related quality of life measures, and this difference may limit the generalizability of results.

Average 2MWD was greater than the arithmetic third of the 6MWD (Table 1), but we are unable to determine from this study how distance walked in the 2MWT compared to distance covered in the first 2 minutes of the 6MWT, nor did we measure oxygen uptake during the tests. Future studies to examine oxygen uptake during the 2MWT in COPD would provide more information about the metabolic cost of this walking test and help identify how the test could be used in exercise prescription.

Further examination of 2MWD responsiveness and MID in other clinical COPD populations is also recommended, including at the time of recovery from hospital admission due to exacerbation or in patients with very low levels of habitual physical activity.

\section{Conclusion}

Improvement of at least $5.5 \mathrm{~m}$ in $2 \mathrm{MWD}$ following a PR program in people with COPD corresponded to a change that was likely to be clinically meaningful, based on anchoring against clinically important change in the 6MWD. Due to the presence of a learning effect, it is recommended that the conduct of the 2MWT includes two trials.

\section{Acknowledgments}

The authors acknowledge the assistance of the Royal Adelaide Hospital Pulmonary Rehabilitation Program staff and study participants in facilitating the conduct of this research. An earlier version of this research was presented at the Annual Scientific Meeting of the Thoracic Society of Australia and New Zealand in Canberra, Australia, on March 27, 2017, and the abstract only of this conference presentation has been previously published.

\section{Disclosure}

The authors report no conflicts of interest in this work.

\section{References}

1. Singh SJ, Puhan MA, Andrianopoulos V, et al. An official systematic review of the European Respiratory Society/American Thoracic Society: measurement properties of field walking tests in chronic respiratory disease. Eur Respir J. 2014;44(6):1447-1478.
2. Holland A, Spruit M, Troosters T, et al. An official European Respiratory Society/American Thoracic Society technical standard: field walking tests in chronic respiratory disease. Eur Respir J. 2014;44(6): $1428-1446$.

3. Steiner M, Holzhauer-Barrie J, Lowe D, et al. Pulmonary Rehabilitation: Steps to Breathe Better. National Chronic Obstructive Pulmonary Disease (COPD) Audit Programme: Clinical Audit of Pulmonary Rehabilitation Services in England and Wales 2015. National Clinical Audit Report. London: Royal College of Physicians; 2016.

4. Osadnik CR, Borges RC, McDonald CF, Carvalho CR, Holland AE. Two 6-minute walk tests are required during hospitalisation for acute exacerbation of COPD. COPD. 2016;13(3):288-292.

5. Borges RC, Carvalho CR. Physical activity in daily life in Brazilian COPD patients during and after exacerbation. COPD. 2012;9(6): 596-602.

6. Brooks D, Davis AM, Naglie G. The feasibility of six-minute and twominute walk tests in in-patient geriatric rehabilitation. Can J Aging. 2007; 26(2):159-162.

7. Brooks D, Parsons J, Tran D, et al. The two-minute walk test as a measure of functional capacity in cardiac surgery patients. Arch Phys Med Rehabil. 2004;85(9):1525-1530.

8. Alfano L, Lowes L, Dvorchik I, et al. The 2-min walk test is sufficient for evaluating walking abilities in sporadic inclusion body myositis. Neuromuscul Disord. 2014;24(3):222-226.

9. Pidala J, Chai X, Martin P, et al. Hand grip strength and 2-minute walk test in chronic graft-versus-host disease assessment: analysis from the chronic GVHD consortium. Biol Blood Marrow Transplant. 2013;19(6):967-972.

10. Eiser N, Willsher D, Dore CJ. Reliability, repeatability and sensitivity to change of externally and self-paced walking tests in COPD patients. Respir Med. 2003;97(4):407-414.

11. Leung AS, Chan KK, Sykes K, Chan KS. Reliability, validity, and responsiveness of a 2-min walk test to assess exercise capacity of COPD patients. Chest. 2006;130(1):119-125.

12. Gloeckl R, Teschler S, Jarosch I, Christle JW, Hitzl W, Kenn K. Comparison of two- and six-minute walk tests in detecting oxygen desaturation in patients with severe chronic obstructive pulmonary disease - a randomized crossover trial. Chron Respir Dis. 2016;13(3):256-263.

13. Global Initiative for Chronic Obstructive Lung Disease. Global Strategy for the Diagnosis, Management, and Prevention of Chronic Obstructive Pulmonary Disease. 2017. Available from: www.goldcopd.org. Accessed 19 May 2017.

14. Williams JE, Singh S, Sewell L, Guyatt G, Morgan M. Development of a self-reported chronic respiratory questionnaire (CRQ-SR). Thorax. 2001;56(12):954-959.

15. Jaeschke R, Singer J, Guyatt G. Measurement of health status. Ascertaining the minimal clinically important difference. Control Clin Trials. 1989;10(4):407-415.

16. Holland A, Hill C, Rasekaba T, Lee A, Naughton M, McDonald C. Updating the minimal important difference for six-minute walk distance in patients with chronic obstructive pulmonary disease. Arch Phys Med Rehabil. 2010;91(2):221-225.

17. Caughey G, Roughead EE, Vitry AI, McDermott RA, Shakib S, Gilbert AL. Comorbidity in the elderly with diabetes: identification of areas of potential treatment conflicts. Diabetes Res Clin Pract. 2010; 87(3):385-393.

18. World Health Organisation Collaborating Centre for Drug Statistics Methodology. ATC/DDD Index 2017. Oslo: 2017. Available from: https://www.whocc.no/atc_ddd_index/. Accessed 19 May 2017.

19. Cohen J. A power primer. Psychol Bull. 1992;112(1):155-159.

20. Jones P. Estimation and application of the minimum clinically important difference in COPD. Lancet Respir Med. 2014;2(3):167-169.

21. Revicki D, Hays R, Cella D, Sloan J. Recommended methods for determining responsiveness and minimally important differences for patient-reported outcomes. J Clin Epidemiol. 2008;61(2):102-109.

22. Schünemann HJ, Puhan M, Goldstein R, Jaeschke R, Guyatt G. Measurement properties and interpretability of the chronic respiratory disease questionnaire (CRQ). COPD. 2005;2(1):81-89. 
23. Beaton D, Boers M, Wells G. Many faces of the minimal clinically important difference (MCID): a literature review and directions for future research. Curr Opin Rheumatol. 2002;14(2):109-114.

24. Pin T. Psychometric properties of 2-minute walk test: a systematic review. Arch Phys Med Rehabil. 2014;95(9):1759-1775.

25. Yuksel E, Kalkan S, Cekmece S, Unver B, Karatosun V. Assessing minimal detectable changes and test-retest reliability of the timed up and go test and the 2-minute walk test in patients with total knee arthroplasty. J Arthroplasty. 2017;32(2):426-430.

26. Hiengkaew V, Jitaree K, Chaiyawat P. Minimal detectable changes of the Berg Balance Scale, Fugl-Meyer Assessment Scale, timed "up \& go" test, gait speeds, and 2-minute walk test in individuals with chronic stroke with different degrees of ankle plantarflexor tone. Arch Phys Med Rehabil. 2012;93(7):1201-1208.

27. Stolwijk-Swuste JM, Beelen A, Lankhorst GJ, Nollet F; CARPA Study Group. SF36 physical functioning scale and 2-minute walk test advocated as core qualifiers to evaluate physical functioning in patients with late-onset sequelae of poliomyelitis. J Rehabil Med. 2008; 40(5):387-394.

28. Holland AE, Nici L. The return of the minimum clinically important difference for 6-minute-walk distance in chronic obstructive pulmonary disease. Am J Respir Crit Care Med. 2013;187(4):335-341.

29. Bohannon RW, Crouch R. Minimal clinically important difference for change in 6-minute walk test distance of adults with pathology: a systematic review. J Eval Clin Pract. 2017;23(2):377-381.
30. Puhan MA, Mador MJ, Held U, Goldstein R, Guyatt GH, Schunemann HJ. Interpretation of treatment changes in 6-minute walk distance in patients with COPD. Eur Respir J. 2008;32(3):637-643.

31. Kamper SJ, Maher CG, Mackay G. Global rating of change scales: a review of strengths and weaknesses and considerations for design. J Man Manip Ther. 2009;17(3):163-170.

32. Schwartz CE, Powell VE, Rapkin BD. When global rating of change contradicts observed change: examining appraisal processes underlying paradoxical responses over time. Qual Life Res. 2017;26(4): 847-857.

33. Schmitt J, Di Fabio RP. The validity of prospective and retrospective global change criterion measures. Arch Phys Med Rehabil. 2005; 86(12):2270-2276.

34. Guyatt GH, Norman GR, Juniper EF, Griffith LE. A critical look at transition ratings. J Clin Epidemiol. 2002;55(9):900-908.

35. Kamper S, Ostelo R, Knol D, Maher C, de Vet H, Hancock M. Global perceived effect scales provided reliable assessments of health transition in people with musculoskeletal disorders, but ratings are strongly influenced by current status. J Clin Epidemiol. 2010;63(7):760-766.

36. Ries AL. Minimally clinically important difference for the UCSD Shortness of Breath Questionnaire, Borg Scale and Visual Analogue Scale. COPD. 2005;2(1):105-110.

37. Cecins N, Landers H, Jenkins S. Community-based pulmonary rehabilitation in a non-healthcare facility is feasible and effective. Chron Respir Dis. 2017;14(1):3-10. 


\section{Supplementary material}

Table SI Baseline characteristics of recruited participants who did and did not complete valid post-PR testing

\begin{tabular}{|c|c|c|c|}
\hline Characteristics & $\begin{array}{l}\text { Valid post PR data } \\
\text { available }(n=4 I) \text {, mean } \\
\text { (SD) or frequency }\end{array}$ & $\begin{array}{l}\text { No valid post PR data } \\
\text { available }(n=3 I) \text {, mean } \\
\text { (SD) or frequency }\end{array}$ & $\begin{array}{l}P \text {-value, test for } \\
\text { between group }^{\text {difference }}\end{array}$ \\
\hline Age, years & $70.5(8.2)$ & $66.9(12.2)$ & 0.17 \\
\hline Male gender & 22 & 17 & 0.92 \\
\hline \multicolumn{4}{|l|}{ Smoking status } \\
\hline Never smoked & 5 & 0 & 0.001 \\
\hline Current smoker & 3 & 12 & \\
\hline Exsmoker & 33 & 19 & \\
\hline \multicolumn{4}{|l|}{ LTOT } \\
\hline Yes & 5 & 5 & 0.63 \\
\hline No & 36 & 26 & \\
\hline FEV $\%$ pred & $51.8(21.0)$ & $41.9(16.7) n=29$ & 0.04 \\
\hline \multicolumn{4}{|l|}{ mMRC } \\
\hline 0 & 2 & 2 & 0.96 \\
\hline 1 & 11 & 6 & \\
\hline 2 & 13 & 10 & \\
\hline 3 & 10 & 9 & \\
\hline 4 & 4 & 3 & \\
\hline Pre-PR 2MWD (test I) & I25.4 (32.3) & I24.3 (25) & 0.89 \\
\hline Pre-PR 6MWD & $327.7(97.9)$ & $298.8(99.4)$ & 0.22 \\
\hline
\end{tabular}

Note: andependent sample t-test (continuous data) or chi square test (categorical data).

Abbreviations: FEV \%pred, percentage predicted forced expiratory volume in I second; LTOT, long-term oxygen therapy; 2MWD, 2-minute walk distance; 6MWD, 6-minute walk distance; mMRC, modified Medical Research Council; PR, pulmonary rehabilitation.

\section{Publish your work in this journal}

The International Journal of COPD is an international, peer-reviewed journal of therapeutics and pharmacology focusing on concise rapid reporting of clinical studies and reviews in COPD. Special focus is given to the pathophysiological processes underlying the disease, intervention programs, patient focused education, and self management protocols.

\section{Dovepress}

This journal is indexed on PubMed Central, MedLine and CAS. The manuscript management system is completely online and includes a very quick and fair peer-review system, which is all easy to use. Visit http://www.dovepress.com/testimonials.php to read real quotes from published authors. 